\title{
DETECTION OF TIME-VARYING SIGNALS IN THE NOISE USING NORMALISED RADIAL BASIS FUNCTION NEURAL NETWORK
}

\author{
Minfen Shen ${ }^{1,2}$, Yuzheng Zhang ${ }^{1}$, K. H. Ting ${ }^{2}$, Francis H. Y. Chan ${ }^{2}$ \\ 1. Science Research Center, Shantou University, Guangdong 515063, China. \\ Email:mfshen@stu.edu.cn \\ 2. Department of Electronic Engineering, Hong Kong University, Hong Kong. \\ Email: fhychan@eee.hku.hk
}

\begin{abstract}
Evoket potentials (EPs) are the special signals that are non-stationary and corrupted by relatively large background noise. To extract the time-varying EP responses more correctly from the noise, a new method is proposed to investigate the problem of denoising the EP signals. The main objective is to estimate the amplitude and the latency without losing the individual properties of each epoch, which is meaningful to clinicians and recognition problems. A normalized radial basis function neural network (NRBFNN) was presented to process the raw EP signals for the purpose of canceling the background noise. The output of NRBFNN enables to effectively track the EPs' variations since the proposed basis functions covers the whole input space with the same degree. Simulations and experimental results confirmed the superior performance of NRBFNN over other methods.
\end{abstract}

Keywords: Evoked potentials (EPs), Time-varying signal, Normalised RBF neural network.

\section{INTRODUCTION}

Evoked potentials are the responses of the ongoing electroencephalographic (EEG) caused by external stimulation. There have existed many evidences that EPs are non-stationary process with very low signal-to noise radio (SNR) and have characteristics that vary across the stimulus trials, which imply a close relationship with the recognition ability of the brain.

The most widely used method of estimating the EPs is the ensemble average (EA) procedure, which ignores the fact that the EP signais are time-varying and fail to track the trial-to trial variations in latency and amplitude. Furthermore, since hundreds to thousands of trials are $0-7803-7702-8 / 03 / \$ 17.00 @ 2003 \mathrm{IEEE}$ needed for EA processing, side effects such as subject tiredness appear that eventually corrupts the average results [1].

To extract the on-line temporal information of EPS and detect the inter-trial variability, a variety of methods have been devised for this purpose. Adaptive noise canceller (ANC) is a method widely used in signal estimation [6]. It has been proved that the improvement of reducing the background noise at the $\mathrm{ANC}$ output is proportional to the SNR of the reference input. Adaptive filter needs a meaningful reference signal for effective tracking. RBFNN provides an unbiased estimate of the EPs, which can be used as a reference signal for the ANC.

Recently, the use of neural networks with built-in nonlinear processing unit has been widely used in many areas. Radial basis function neural network (RBFNN) can be directly used to deal with the nonlinear multi-dimensional continuous functions and to track the single trial EPs since it has the universal approximation capability [2-3].

However, when we meet the signals with low SNR, the methods mentioned above fail to extract the time-varying EP signals effectively. To track the signals variations with good SNR improvement, a normalized RBFNN scheme is proposed in this paper. This novel method can be achieved to out-perform the methods based on RBFNN or the nonlinear ANC with RBFNN prefilter.

\section{NORMALISED RBFNN}

The structure of a common RBFNN is shown in Fig. 1 which is a multiplayer feed-forward neural network consisting of input layer, kernel (hidden) layer and output layer. The units in the kernel layer provide an array of nonlinear radial basis functions, which are frequently selected as Gaussian functions. The radial basis function (RBF) network is defined 


$$
y=f(x)=\sum_{i=1}^{M} w_{i} \phi_{i}\left(x_{i} ; c_{i} \sigma_{i}\right)
$$

The radial basis functions $\phi_{i}$ are set as

$$
\phi_{i}\left(x_{i} ; c_{i}, \sigma_{i}\right)=\exp \left(\frac{-\left(x_{i}-c_{i}\right)^{2}}{\sigma_{i}^{2}}\right)
$$

where $x$ is the input vector, $c_{i}$ is the vector determining the center of the basis function associated with kemel unit $i$. $w_{i}$ represents the weight from kernel unit to output unit. In the output layer, the value of unit is obtained through a linear combination of the nonlinear outputs from the kernel layer. $\sigma_{i}$ denotes the widths of the basis functions. The weights are trained in a supervised fashion using an appropriate linear learning method.

Normalization of the basis function activations in a RBF network is a common way for achieving the partition of unity desired for practical modeling. It results in the basis functions covering the whole input space in the same degree. Partitioning of unity is an important property for basis function networks in many applications.

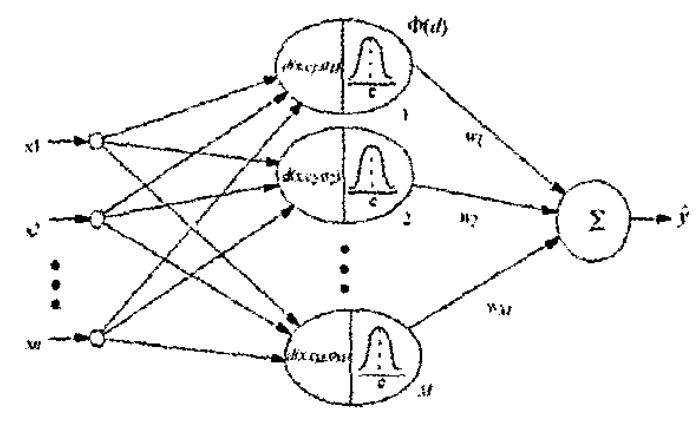

Fig.1.The structure diagram of RBFNN.

Since RBFNN can be less sensitive to poor center selection and in cases where the network is being used within a local model structure, a partition of unity is highly desirable [3]. The RBF is defined as:

$$
\Phi_{i}=\frac{\phi_{i}}{\sum_{j=1}^{M} \phi_{j}}
$$

The neural networks can form a partition of unity to improve the interpolation properties and makes the network less sensitive to the choice of the width $\sigma_{i}$ due to the normalization [4-5].

Normalization of the basis functions in such a network is often motivated by expecting to achieve a partition of unity across the input space. By partition of unity, the sum of the normalized basis functions equals unity at any point in the input space

$$
\sum_{i=1}^{M} \vec{\phi}_{k}(x)=1
$$

Unlike the common cases where points were given different weightings, the normalised RBFNN has the effect of covering every point of the input space to the same degree.

\section{PERFORMANCE EVALUATION}

In the following examples, the performance of the three methods presented above are tested. 600 response trials of visual evoket potential (VEPs) are averaged to generate the pure input. Gaussian white noise was added to the input to generate 50 epochs with 500 samples. The input SNR is set with $-40 \mathrm{~dB}$.

The performance based on NRBFNN was compared with the methods of ANC with RBFNN prefilter and RBFNN, as shown in Fig.2. To evaluate the performance of noise reduction, the relative mean square error (MSE) is defined as

$$
M S E=\frac{E\left[(s-y)^{2}\right]}{E\left[s^{2}\right]}
$$

Where $y$ represents the output of the system and $s$ denotes the primary VEP input. The performance for three methods is evaluated by estimating the MSE and shown in terms of the MSE versus different trials in Fig. 3 with $-40 \mathrm{~dB}$ signal input.

Obviously, in terms of MSE, NRBFNN is found to be far better as compared to the methods of both ANC with RBFNN prefilter and RBFNN. The results indicated that different abilities for the EEG noise reduction with different trials. The NRBFNN scheme significantly increases the ability of tracking the time-varying VEP signals with very low SNR. The normalized RBFNN has the best performance in the fitting ability, as well as in the rate of convergence, in all situations. 


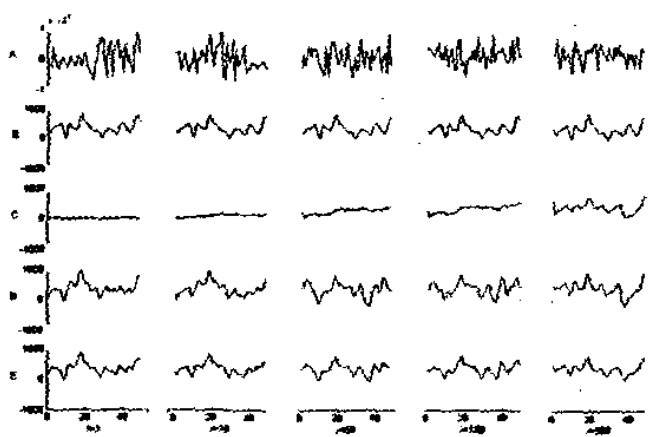

Fig.2. Performance comparison of signal with $-40 \mathrm{~dB}$. indicates the trial number. (A) The primary noisy signal (SNR $=-40 \mathrm{~dB}$ ). (B) The pure VEP signal. (C) the output of the nonlinear ANC. (D) The results using RBFNN. (E) The output based on NRBFNN.

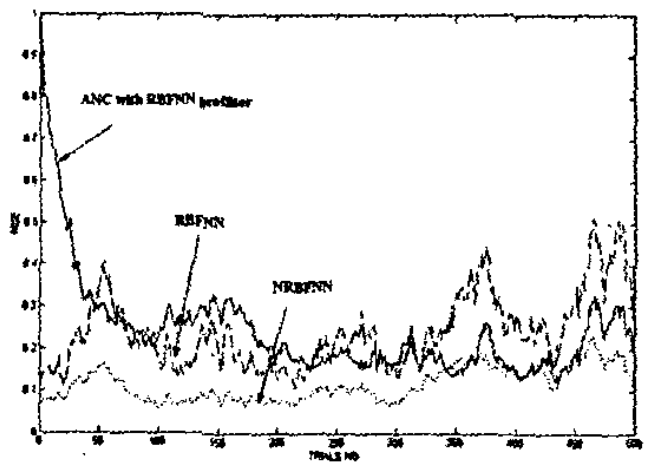

Fig. 3. Comparison of the MSE for three approaches with $-40 \mathrm{~dB}$ input.

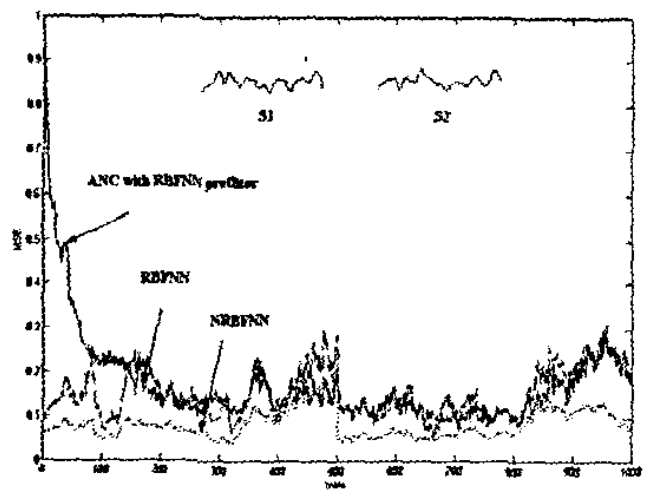

Fig. 4. A comparison of error for three approaches in an abrupt VEP changing simulation with $S N R=-40 \mathrm{~dB}$. Insets are two simulated VEP signals: one is in the first 500 trials and another one is in the last 500 trials.

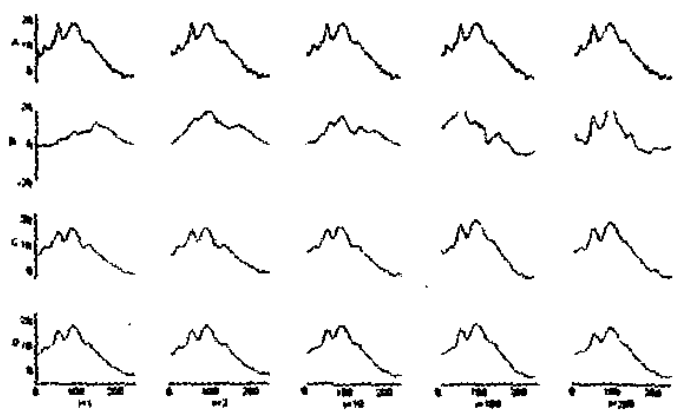

Fig. 5. Tracing the real ERP in different methods (i indicates the trial number), (A) Pure VEP responding to the $\mathrm{O}$-shaped stimuli (obtained by averaging 200 trials); (B) Output of the ANC with RBFNN prefiltering; (C) Output of RBFNN; (D) Results of NRBFNN.
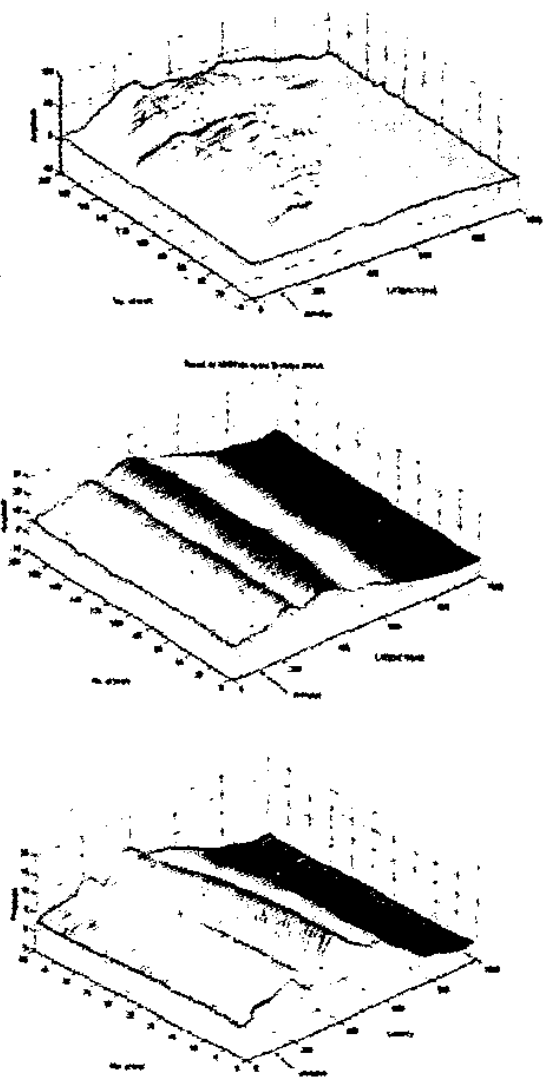

Fig. 6. ERP from O-shape stimuli by the ANC with RBFNN (top); ERP from O-shape stimuli by the NRBFNN (middle); ERP from $X$-shape stimuli by the NRBFNN (below).

The performance of tracking VEPs' variation across trials is also investigated. Abrupt change of recovered VEP signal is generated. Among 1000 trials input signals, the first 500 trials remain the same waveform $S 1$, and other 500 trials is a different 52 , forming a jump in the 500th 
trial. With such underlying VEPs, different amplitude of noise is putting in to produce a primary data with $-40 \mathrm{~dB}$.

The three approaches of VEP extraction described above are again employed to compare the ability of tracking the abrupt. For the ANC with RBFNN prefilter, it reveals that more trials must be needed to achieve a reliable estimation of VEPS under recordings with poor SNR. It can be seen from the Fig. 4 that the NRBFNN algorithm can best track the signal variations. The MSE is significantly greater than that of either RBFNN alone or ANC with RBFNN prefilter.

Finally, event-related potentials (ERPs) are transient changes in the electroencephalogram (EEG) due to external stimuli or cognitive processes triggered by external events. ERPs have found numerous applications in clinical neurophysiology and psychiatry. Similar to EP, averaging is the most popular method for tracking peaks. In this section, NRBFNN is conducted to estimate visual ERPs from actual recordings and to identify how the brain gets to know the visual stimuli by providing a direct comparison of visual response from multiple cortical areas of individual. Comparison among all the three approaches proposed is shown in Fig. 5, together with the grand averaged ERP waveforms calculated. Based on the recordings from $100 \mathrm{~ms}$ stimuli onset to $900 \mathrm{~ms}$ post-onset of the $72 \mathrm{th}$ electrode, NRBFNN deals with the differences between trials. Obviously, the results of NRBFNN and RBFNN are similar with that of the EA method. We must also notice that it is only one trial input by which NRBFNN can make similar results as EA with 200 trials. Fig.6 gives all trials with ANC and NRBFNN. There is no stable waveform by nonlinear ANC, while NRBFNN provides the more perfect results. Thus, we can get the different response in only one trial input and the trial-to-trial variance.

\section{CONCLUSION}

The problem of detecting the time-varying signal in the background noise is very important in many practical applications. Traditional Ensemble Averaging approach exists many drawbacks for tracking the change characteristics of noisy signals. We have proposed an improved method based on normalized radial basis neural network and focused on its application to the extraction of the real VEP signal with low SNR. The variance between trials is meaningful to clinicians and recognition problems. It has been shown that the NRBFNN is more applicable to the real-time detection of single-trial VEPs than other methods such as the nonlinear ANC with RBFNN prefilter or the RBFNN alone. After normalization, the local interpolation properties are significantly improved, which makes the network less sensitive to the choice of the other parameters that made NRBFNN be the best method in terms of latency estimation as well as SNR output. The performance of the proposed methods is also evaluated with MSE and the ability of tracking the primary signals. The experimental results provide convergent evidence that the NRBFNN can effectively attenuate the noise and successfully identify the variance between trials.

Evidence has been provided that our method was superior in tracking the single-trial VEP, compared with other two approaches of both ANC with RBFNN prefilter and RBFNN procedure.

\section{ACKNOWLEDGEMENT}

This work was supported in part by the National Natural . Science Foundation of China (60271023), the Key Grant of Natural Science Foundation of Guangdong, China (021264) and the Key Grant of the Education Ministry of China (02110).

\section{REFERENCES}

[1] S. Nishida, Method for Single-Trial Recording of Somatosensory Evoket Potentials, J. Biomed. Eng., Vol. 15, pp.257-262,1993.

[2] E. J. Hartman, Layered Neural Networkwith Gaussian Hidden Units as Universal Approximation, Neural Computation, Vol. 2, pp.210-215, 1990.

[3] C. F. Zhu, Estimation of Single-Trial Evoked Potential With RBFNN, International Conference on Information Systems Analysis and Synthesis, 2001.

[4] R. Shorten and R. Murray-Smith, On Normalising Basis Function Networks, Proceedings 4th Irish Neural Networks Conference. Dublin, Sept. 1994.

[5] T. Poggio and F. Girosi, Networks for Approximation and Learning, Proceedings of the IEEE: Special Issue on Neural Networks 1, vol. 78, No. 9, Sept. 1990

[6] B. Widrow, Adaptive Noise Canceling: Principles and Applications, Proceedings of IEEE, Vol.63, pp.1692-1716, Dec. 1975.

[7] N. Müller, Closed-Loop Ignition Control Using On-line Learning of Locally-Tuned Radial Basis Function Networks, Proceedings of American Control Conference (ACC), San Diego, USA, June 1999. 